\title{
SU(2) vacuum dynamics in applied external magnetic field
}

Paolo Cea and Leonardo Cosmai ${ }^{\mathrm{a}}$

${ }^{a}$ INFN - Sezione di Bari - Via Amendola 173 - 70126 Bari - Italy

The vacuum dynamics of SU(2) lattice gauge theory is studied by means of a gauge-invariant effective action, both at zero and finite temperature. Working with lattices up to $32^{4}$ we check the scaling of the energy density with the magnetic length. We find that the screening at zero temperature of the applied external magnetic field weakens by increasing the temperature.

\section{INTRODUCTION}

The aim of this work is to study the nonperturbative vacuum dynamics of the SU(2) 1.g.t. in presence of a static external applied field. To this purpose we use a gauge-invariant effective action for the external background field defined by means of the lattice Schrödinger functional[12]

$\mathcal{Z}\left[\vec{A}^{\mathrm{ext}}\right]=\int \mathcal{D} U \exp \left(-S_{W}\right)$,

where $S_{\mathrm{W}}$ is the Wilson action and $\overrightarrow{A^{\mathrm{ext}}}(\vec{x})=$ $\vec{A}_{a}^{\text {ext }}(\vec{x}) \lambda_{a} / 2$ is the external field. We adopt periodic boundary conditions in space and time direction with the integration constraint over the lattice links

$$
\begin{aligned}
& \left.U_{\mu}(x)\right|_{x_{4}=0}=U_{\mu}^{\mathrm{ext}}(\vec{x}, 0) \\
& =\mathrm{P} \exp \left\{+i a g \int_{0}^{1} d t A_{\mu}^{\mathrm{ext}}(x+a t \hat{\mu})\right\} .
\end{aligned}
$$

The Schrödinger functional is invariant under arbitrary lattice gauge transformations of the boundary links. The lattice effective action for the background field $A_{\mu}^{\mathrm{ext}}(\vec{x})$ is defined by means of the lattice Schrödinger functional Eq. (1) (T extension in Euclidean time):

$\Gamma\left[\vec{A}^{\mathrm{ext}}\right]=-\frac{1}{T} \ln \left\{\frac{\mathcal{Z}\left[U^{\mathrm{ext}}\right]}{\mathcal{Z}[0]}\right\}$.

$\mathcal{Z}[0]$ is the lattice Schrödinger functional without external background field $\left(U_{\mu}^{\text {ext }}=1\right)$. In the continuum limit $(T \rightarrow \infty) \Gamma\left[\vec{A}^{\text {ext }}\right]$ is the vacuum energy in presence of the background field $\vec{A}^{\text {ext }}(\vec{x})$. Our gauge-invariant effective action can be used for a non-perturbative investigation of the properties of the SU(2) l.g.t. vacuum.
We consider static background fields that give rise to constant field strength. In this case $\Gamma\left[\vec{A}^{\text {ext }}\right]$ is proportional to the spatial volume $V$ and the relevant quantity is the density of the effective action:

$\varepsilon\left[\vec{A}^{\mathrm{ext}}\right]=-\frac{1}{\Omega} \ln \left[\frac{\mathcal{Z}\left[U^{\mathrm{ext}}\right]}{\mathcal{Z}(0)}\right], \quad \Omega=V \cdot T$.

In particular we consider an external constant abelian magnetic field:

$\vec{A}_{a}^{\mathrm{ext}}=\delta_{a, 3} \vec{A}^{\mathrm{ext}}, A_{k}^{\mathrm{ext}}=\delta_{k, 2} x_{1} H, F_{12}^{a}=\delta^{a, 3} H$.

On the lattice

$U_{2}^{\mathrm{ext}}(x)=\cos \left(\frac{a g H x_{1}}{2}\right)+i \sigma^{3} \sin \left(\frac{a g H x_{1}}{2}\right)$

$U_{1}^{\text {ext }}(x)=U_{3}^{\text {ext }}(x)=U_{4}^{\text {ext }}(x)=1$.

Periodic boundary conditions imply the quantization of the magnetic field:

$\frac{a^{2} g H}{2}=\frac{2 \pi}{L_{1}} n_{\mathrm{ext}}, \quad n_{\mathrm{ext}} \quad$ integer .

We want to compute the vacuum energy density Eq.(4). To avoid the problem of dealing with a partition function we focus on $\varepsilon^{\prime}\left[\vec{A}^{\text {ext }}\right]$, the derivative of $\varepsilon\left[\vec{A}^{\mathrm{ext}}\right]$ with respect to $\beta$, by taking $n_{\mathrm{ext}}$ (i.e. $g H$ ) fixed $[3] . \quad \varepsilon\left[\vec{A}^{\text {ext }}\right]$ can be obtained by a numerical integration in $\beta$.

We performed lattice simulations on $32 \times L^{2} \times 32$ lattices ( $\mathrm{L}=6,8,10,16,20,24,32)$ with periodic boundary conditions using the Quadrics/QH1 in Bari. The links belonging to the time slice $x_{4}=0$ and to the spatial boundary are constrained according to Eq. (6). The constraint on the links starting from sites belonging to the spatial boundary corresponds in the continuum to the usual requirement that the fluctuations over the background field vanish at the spatial infinity. 
$32 \times L^{2} \times 32 \quad(L=32)$

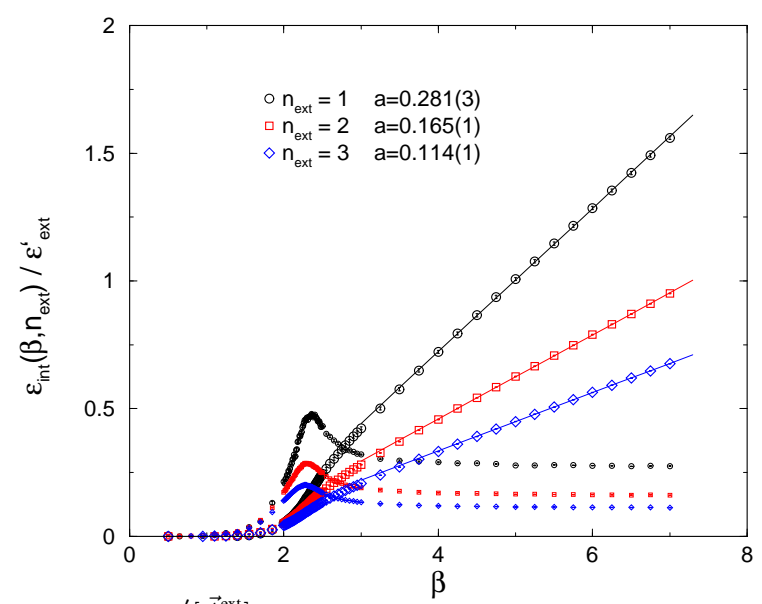

Figure 1. $\frac{\varepsilon^{\prime}\left[\vec{A}^{\text {ext }}\right]}{\varepsilon_{\text {ext }}^{\prime}}$ versus $\beta$ on a $32^{4}$ lattice with the result of the numerical integrations (solid lines).

Therefore the contributions to $\varepsilon^{\prime}\left[\vec{A}^{\mathrm{ext}}\right]$ due to the constrained links must be subtracted and we have to measure the derivative of the "internal" energy density $\varepsilon_{\text {int }}^{\prime}$ obtained by evaluating the contribution to $\varepsilon^{\prime}\left[\overrightarrow{A^{\mathrm{ext}}}\right]$ given by the dynamical links. We numerically integrate the data and obtain (see Fig 1 for $\beta \gg 1$ :

$\varepsilon_{\mathrm{int}}\left(\beta, n_{\mathrm{ext}}\right) \simeq a\left(n_{\mathrm{ext}}\right) \frac{H^{2}}{2} a\left(n_{\mathrm{ext}}\right) \beta$,

where $a\left(n_{\text {ext }}\right) \ll 1$ to be contrasted[3] to the case of $\mathrm{U}(1)$ where $a\left(n_{\text {ext }}\right) \simeq 1$.

\section{THE SU(2) VACUUM ENERGY DENSITY}

The data for $\varepsilon_{\text {int }}^{\prime}\left(\beta, n_{\text {ext }}\right) / \varepsilon_{\text {ext }}^{\prime}$ at the perturbative tail and at the peak for various lattice sizes and values of $n_{\text {ext }}$ can be expressed as a function of the scaling variable $x=a_{H} / L_{\text {eff }}$, where $a_{H}=\sqrt{2 \pi / g H}=$ $\sqrt{L_{1} /\left(2 n_{\text {ext }}\right)}$ is the magnetic length and $L_{\text {eff }}=\Omega_{\text {int }}^{1 / 4}$ is the lattice effective linear size.

At fixed $\beta$, we can fit these data to a power-law behavior:

$\frac{\varepsilon_{\mathrm{int}}^{\prime}\left(\beta, n_{\mathrm{ext}}\right)}{\varepsilon_{\mathrm{ext}}^{\prime}}=k(\beta) x^{\alpha}$.

This suggests that the lattice data scale according to

$\frac{\varepsilon_{\text {int }}^{\prime}\left(\beta, n_{\mathrm{ext}}, L_{\mathrm{eff}}\right)}{\varepsilon_{\mathrm{ext}}^{\prime}}=k(\tilde{\beta}) x^{\alpha}, \quad \alpha=1.5$,
$32 \times L^{2} \times 32 \quad(L=16,24,32)$

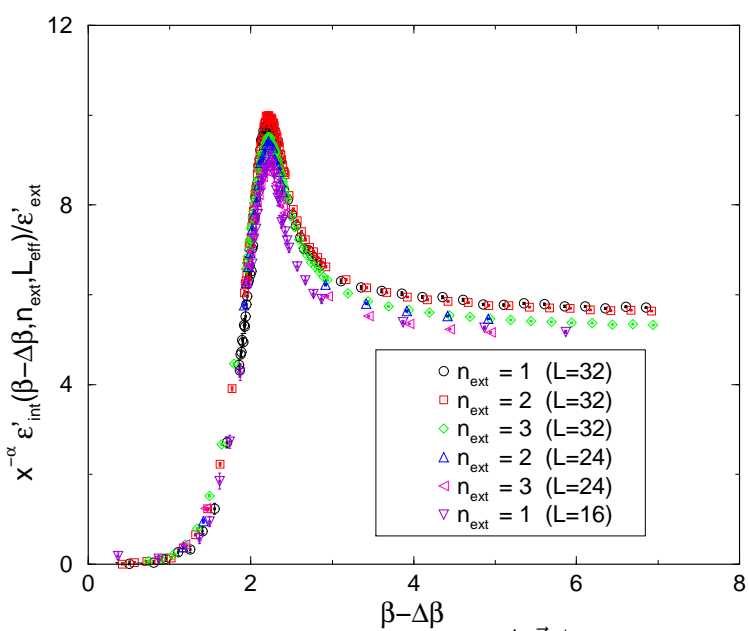

Figure 2. The universality plot for $\frac{\varepsilon^{\prime}\left[\vec{A}^{\text {ext }}\right]}{\varepsilon_{\text {ext }}^{\prime}}$ versus $\beta-$ $\Delta \beta$.

where $\tilde{\beta}=\beta-\Delta \beta$ and $\Delta \beta$ takes care of the shift of the peak position of $\varepsilon_{\text {int }}^{\prime}$. Indeed in Fig 2 we see that all our numerical data (for all values of $a_{H}$ and $L_{\text {eff }}$ ) can be approximately arranged on the scaling curve $k(\tilde{\beta})$ (Eq.9. Our numerical data suggests that

$\lim _{L_{\text {eff }} \rightarrow \infty} \varepsilon_{\text {int }}\left(\beta, n_{\text {ext }}, L_{\text {eff }}\right)=0$.

in the whole range of $\beta$. This would imply that in the continuum limit $\left(L_{\text {eff }} \rightarrow \infty\right), \beta \rightarrow \infty$ the SU(2) vacuum screens completely the external chromomagnetic abelian field, behaving like a abelian magnetic condensate medium.

\section{FINITE TEMPERATURE}

We also studied the SU(2) gauge system in an external chromomagnetic abelian field at finite temperature in order to investigate if a connection exists between the external chromomagnetic field and confinement. We looked at the behavior of the temporal Polyakov loop vs. the external applied field. We considered a $\mathrm{SU}(2)$ gauge system at $\beta=2.5$ on a $32^{3} \times 5$ lattice at zero applied external (i.e. $n_{\mathrm{ext}}=0$ ) which is in the deconfined phase of finite temperature $\mathrm{SU}(2)$. If the external field strength is increased the expectation value of the Polyakov loop is driven towards the value at zero temperature (see Fig 3). A similar observation has been reported in Ref. [4]. On 
$32^{3} \times 5$

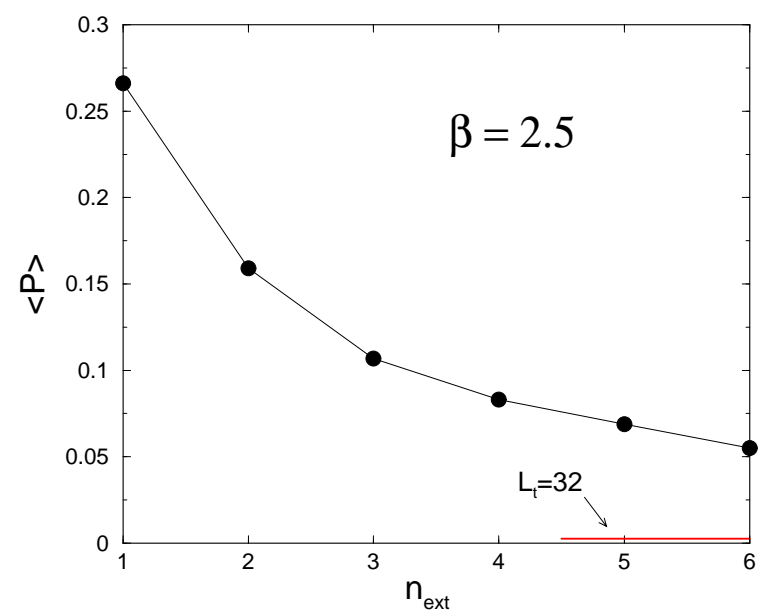

Figure 3. The temporal Polyakov loop vs. $n_{\text {ext }}$.

the other hand, if we start with a SU(2) gauge system at zero temperature in a constant abelian chromomagnetic background field of fixed strength $\left(n_{\mathrm{ext}}=1\right)$ and increase the temperature the perturbative tail of the $\beta$ derivative of the energy density $\varepsilon_{\text {int }}^{\prime}\left(\beta, n_{\text {ext }}\right) / \varepsilon_{\text {ext }}^{\prime}$ increases with $1 / L_{t}$ and tends towards the "classical" value $\varepsilon_{\text {int }}^{\prime}\left(\beta, n_{\text {ext }}\right) / \varepsilon_{\text {ext }}^{\prime}=1$ We may conclude that increasing the temperature there is no screening effect for the energy density, confirming that the zerotemperature screening of the external field is related to the confinement.

Moreover the information on $\varepsilon_{\text {int }}^{\prime}\left(\beta, n_{\mathrm{ext}}\right) / \varepsilon_{\mathrm{ext}}^{\prime}$ at finite temperature can be used to get an estimate of $T_{c} / \Lambda_{\text {latt }}$. Our data can be parameterized near the peak as

$\frac{\varepsilon_{\text {int }}^{\prime}\left(\beta, L_{t}\right)}{\varepsilon_{\text {ext }}^{\prime}}=\frac{a_{1}\left(L_{t}\right)}{a_{2}\left(L_{t}\right)\left[\beta-\beta^{*}\left(L_{t}\right)\right]^{2}+1}$.

So that we get an estimate of the deconfinement temperature (see Fig.4):

$$
\begin{gathered}
\frac{T_{c}}{\Lambda_{\text {latt }}}=\frac{1}{L_{t}} \frac{1}{f\left(\beta^{*}\left(L_{t}\right)\right)}=27.12(4.04) \\
f(\beta)=\left(\frac{11}{6 \pi^{2}} \frac{1}{\beta}\right)^{-\frac{51}{121}} \exp \left(-\frac{3 \pi^{2}}{11} \beta\right)
\end{gathered}
$$

Our result is consistent with previous studies [5].

\section{CONCLUSIONS}

We have studied the non-perturbative dynamics of the SU(2) 1.g.t. vacuum in an external abelian chro-

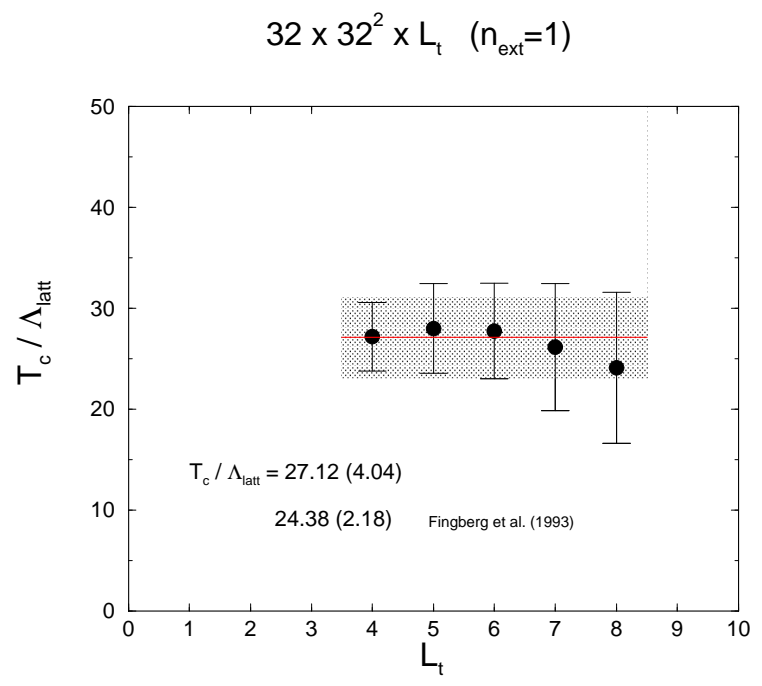

Figure 4. $T_{c} / \Lambda_{\text {latt }}$ vs. $L_{t}$.

momagnetic field, by means of a gauge invariant effective action defined using the lattice Schrödinger functional.

Our numerical results suggests that in the continuum limit ( $L_{\text {eff }} \rightarrow \infty, \beta \rightarrow \infty$ ) the energy density $\varepsilon_{\text {int }}\left(\beta, L_{\text {eff }}, n_{\text {ext }}\right) \rightarrow 0$, i.e.: the $\mathrm{SU}(2)$ vacuum screens completely the external abelian chromomagnetic field and behaves like an abelian magnetic condensate in agreement with the dual superconductor scenario for the color confinement.

At finite temperature it seems that confinement is restored by increasing the strength of the external applied field. On the other hand the zero temperature screening of the external field is removed by increasing the temperature.

\section{REFERENCES}

1. G. C. Rossi and M. Testa, Nucl. Phys. B163 (1980) 109; ibid. B176 (1980) 477.

2. M. Lüscher, R. Narayanan, P. Weisz, and U. Wolff, Nucl. Phys. B384 (1992) 168; M. Lüscher and P. Weisz, Nucl. Phys. B452 (1995) 213.

3. P. Cea, L. Cosmai, and A. D. Polosa, Phys. Lett. B392 (1997) 177; Phys. Lett. B397 (1997) 229; P. Cea, L. Cosmai, Mod. Phys. Lett. A13 (1998) 861.

4. M. Ogilvie, Nucl. Phys. B (Proc.Suppl) 63 (1998) 430.

5. J. Fingberg, U. Heller, F. Karsch, Nucl. Phys. B392 (1993) 493. 